\title{
A NEW PROOF OF A NORDGREN, ROSENTHAL AND WINTROBE THEOREM ON UNIVERSAL OPERATORS
}

\author{
CARL C. COWEN AND EVA A. GALLARDO-GUTIÉRREZ
}

\begin{abstract}
A striking result by Nordgren, Rosenthal and Wintrobe states that the Invariant Subspace Problem is equivalent to the fact that any minimal invariant subspace for a composition operator $C_{\varphi}$ induced by a hyperbolic automorphism $\varphi$ of the unit disc $\mathbb{D}$ acting on the classical Hardy space $H^{2}$ is one dimensional. We provide a completely different proof of Nordgren, Rosenthal and Wintrobe's Theorem based on analytic Toeplitz operators.
\end{abstract}

\section{INTRODUCTION}

In the eighties, Nordgren, Rosenthal and Wintrobe [7] gave an equivalent formulation of the longstanding open question known as the Invariant Subspace Problem stated in terms of composition operators acting on the classical Hardy space $H^{2}$. In particular, they showed that if $\varphi$ is a hyperbolic automorphism of the complex unit disc $\mathbb{D}$, then every bounded operator on a complex Hilbert space of dimension greater than one has a nontrivial invariant subspace if and only if the minimal nontrivial invariant subspaces for the composition operator induced by $\varphi$ in $H^{2}$

$$
C_{\varphi} f=f \circ \varphi, \quad\left(f \in \mathcal{H}^{2}\right)
$$

are one dimensional.

Indeed, Nordgren, Rosenthal and Wintrobe proved that $C_{\varphi}-\lambda I$ acting on the Hardy space $H^{2}$ is a universal operator (in the sense of Rota) for any $\lambda$ in the interior of the spectrum of $C_{\varphi}$. Recall that $U$ is universal in the sense of Rota acting on Hilbert space $H$ if for each non-zero bounded operator $A$, there is an invariant subspace $M$ for $U$ and a non-zero number $\mu$ such that $\mu A$ is similar to $\left.U\right|_{M}$, that is, there is a linear isomorphism $X$ of $H$ onto $M$ such that $U X=\mu X A$ (see [9]).

Universal operators in the sense of Rota have attracted the interest of many operator theorists. The main reason for that is clear: the lattice of its invariant subspaces has such a rich structure that they model every operator on a separable infinite dimensional Hilbert space. There are several well-known examples of universal operators in the literature: the adjoint of the shift of infinity multiplicity, adjoints of analytic Toeplitz operators, or operators that have a reducing subspace on which the operator is universal and unitarily equivalent to the adjoint of an analytic Toeplitz operator. For more about the subject, we refer to Chapter 8 of the recent monograph by Chalendar and Partington [2].

Date: January 2016.

1991 Mathematics Subject Classification. Primary 47B38.

Key words and phrases. Universal operators, Composition operators, analytic Toeplitz operators.

Authors are partially supported by Plan Nacional I+D grant no. MTM2013-42105-P. 
Needless to say, proving that a given operator with a rich lattice of invariant subspaces is universal is not an easy task. In 1969, Caradus proved a sufficient condition to provide universal operators on Hilbert spaces (see [1, p. 527]). The best known examples of universal operators, including the operator Rota used to introduce the concept, satisfy the hypotheses of Caradus' Theorem.

Caradus Theorem. If $H$ is a separable Hilbert space and $U$ is a bounded, linear operator on $H$ such that:

(i) The null space of $U$ is infinite dimensional.

(ii) The range of $U$ is $H$.

Then $U$ is universal for $H$.

In this respect, what it was shown by Nordgren, Rosenthal and Wintrobe in [7] is that $C_{\varphi}-\lambda I$ acting on $H^{2}$ satisfies Caradus Theorem for any $\lambda$ in the interior of the spectrum of $C_{\varphi}$. The heart of the proof deals with the ontoness of $C_{\varphi}-\lambda I$ on $H^{2}$, and it relies on a key description of an orthogonal decomposition of $\mathcal{H}^{2}$. The orthogonal decomposition is based on the fact that Blaschke products induce isometric multiplication operators in $H^{2}$ (Wold's Theorem), and considering those whose zero sequence is $\left\{\varphi_{n}\left(z_{0}\right)\right\}_{n \in \mathbb{Z}}$ for $z_{0} \in \mathbb{D}$, it is possible to describe precisely the subspaces in the orthogonal decomposition.

In this note, we present a different proof of Nordgren, Rosenthal and Wintrobe's Theorem by means of analytic Toeplitz operators, avoiding the orthogonal decomposition of $H^{2}$ and the fact that Blaschke products induce isometric multiplication operator in $H^{2}$. Let us point out that other simplifications in the literature of the proof of Nordgren, Rosenthal and Wintrobe's Theorem in the Hardy space seem to be unsuccessful, as Professor Jonathan R. Partington informed us (see [8]).

For the sake of self-completeness, in what follows we recall some preliminaries and prove Nordgren, Rosenthal and Wintrobe's Theorem in Section 2.

1.1. Preliminaries. In [4], the authors showed that the composition operators on $H^{2}$ whose symbols are hyperbolic automorphisms of the unit disk fixing \pm 1 comprise a one-parameter group which is similar to the adjoint of the analytic Toeplitz operators coming from covering maps of annuli centered at the origin whose radii are reciprocals. More precisely, consider the set (for $-\infty<t<\infty$ ) of composition operators with symbols $C_{\varphi_{t}}$ where

$$
\varphi_{t}(z)=\frac{\left(1+e^{-t}\right) z+\left(1-e^{-t}\right)}{\left(1-e^{-t}\right) z+\left(1+e^{-t}\right)}
$$

which has fixed points at 1 and -1 and derivatives at these fixed points $e^{-t}$ and $e^{t}$. Thus, for $t>0$, the Denjoy-Wolff point of $\varphi_{t}$ is 1 . Easy computations show that this is a one-parameter group of operators, $C_{\varphi_{t}} C_{\varphi_{s}}=C_{\varphi_{s+t}}$, and it is not too difficult to see that this group is strongly continuous. Moreover, the infinitesimal generator $H$ is given by

$$
H f(z)=f^{\prime}(z) \frac{1-z^{2}}{2}
$$


which is a closed operator with domain $\left\{f \in H^{2}: f^{\prime}(z)\left(1-z^{2}\right) \in H^{2}\right\}$. In addition, a straightforward computation shows that the function $w_{\lambda}$

$$
w_{\lambda}(z)=\left(\frac{1+z}{1-z}\right)^{\lambda}=\left(\frac{1-z}{1+z}\right)^{-\lambda}, \quad(z \in \mathbb{D}),
$$

is an eigenvector of the infinitesimal generator corresponding to $\lambda$ and it is in $H^{2}$ for $-1 / 2<\operatorname{Re} \lambda<$ $1 / 2$. Note that the eigenspaces of the infinitesimal generator are one-dimensional. Use of the theory of semigroups or a direct computation from the expressions for $\varphi_{t}$ and $w_{\lambda}$ shows that $C_{\varphi_{t}} w_{\lambda}=e^{\lambda t} w_{\lambda}$. In particular, for $t>0$, the point spectrum of $C_{\varphi_{t}}$ is

$$
\sigma_{p}\left(C_{\varphi_{t}}\right)=\left\{\lambda: e^{-t / 2}<|\lambda|<e^{t / 2}\right\} .
$$

On the other hand, the analytic Toeplitz operators whose symbols are maps of the disk onto annuli centered at the origin with radii that are reciprocals of each other, that is, the same family of annuli as occur above as the spectra of the composition operators, are given by

$$
\psi_{t}(z)=e^{\left(\frac{t i}{\pi} \log \left(\frac{1-z}{1+z}\right)\right)}=\left(\frac{1-z}{1+z}\right)^{\frac{t i}{\pi}}
$$

where we choose a normalization so that $t=1$ corresponds to the annulus $\left\{\zeta: e^{-1 / 2}<|\zeta|<e^{1 / 2}\right\}$.

These Toeplitz operators also form a strongly continuous group, $T_{\psi_{t}} T_{\psi_{s}}=T_{\psi_{s+t}}$, and the infinitesimal generator is given by

$$
(G h)(z)=\frac{i}{\pi} \log \left(\frac{1-z}{1+z}\right) h(z)
$$

for $h$ in $H^{2}$. That is, the infinitesimal generator, $G$, of the group is an (unbounded) analytic Toeplitz operator. As is well known, the kernel functions for evaluation at $\alpha$ in the disk, $K_{\alpha}(z)=(1-\bar{\alpha} z)^{-1}$, are eigenvectors for adjoints of analytic Toeplitz operators

$$
G^{*} K_{\alpha}=-\frac{i}{\pi} \log \left(\frac{1-\bar{\alpha}}{1+\bar{\alpha}}\right) K_{\alpha}
$$

and we see, also in this case, that the eigenspaces are one dimensional. We also have

$$
T_{\psi_{t}}^{*} K_{\alpha}=\overline{\psi_{t}(\alpha)} K_{\alpha}=\left(\frac{1-\bar{\alpha}}{1+\bar{\alpha}}\right)^{-\frac{t i}{\pi}} K_{\alpha}
$$

For $-1 / 2<\operatorname{Re} \lambda<1 / 2$, a computation shows that

$$
w_{\lambda}=\left(\frac{1-z}{1+z}\right)^{-\lambda} \quad \text { and } \quad v_{\lambda}=\left(1-\frac{-i \sin \left(\lambda \frac{\pi}{2}\right)}{\cos \left(\lambda \frac{\pi}{2}\right)} z\right)^{-1}
$$

are eigenvectors that correspond to the eigenvalue $\lambda$ for the infinitesimal generators $H$ and $G$, respectively (see [4]). By matching up such eigenvectors, the authors prove the following theorem which will be the main tool in Section 2.

Theorem ([4, Theorem 5]). Let the vectors $w_{\lambda}$ and $v_{\lambda}$ be as described in Equation (1) for $-1 / 2<$ $\lambda<1 / 2$. Then the following are true:

(i) The sets $\left\{w_{\lambda}:-1 / 2<\lambda<1 / 2\right\}$ and $\left\{v_{\lambda}:-1 / 2<\lambda<1 / 2\right\}$ are each linearly independent and have dense span in $H^{2}$. 
(ii) If $S$ is the operator obtained from

$$
S\left(v_{\lambda}\right)=\frac{1}{\sqrt{2}} w_{\lambda}
$$

defining it to be linear from the span of $\left\{v_{\lambda}:-1 / 2<\lambda<1 / 2\right\}$ to the span of $\left\{w_{\lambda}:-1 / 2<\right.$ $\lambda<1 / 2\}$, then $S$ can be extended to be a bounded operator of $H^{2}$ onto itself with bounded inverse.

(iii) The operator $S$ of Equation ( $\star$ ) gives a similarity of the one-parameter groups $\left\{C_{\varphi_{t}}\right\}_{t \in \mathbb{R}}$ and $\left\{T_{\psi_{t}}^{*}\right\}_{t \in \mathbb{R}}$. In particular, for each real number $t$,

$$
C_{\varphi_{t}} S=S T_{\psi_{t}}^{*}
$$

With this theorem at hand, we are in position to show a Toeplitz operator based on proof of Nordgren, Rosenthal and Wintrobe's Theorem on universality of $C_{\varphi}-\lambda I$.

\section{A Toeplitz operator proof of a Nordgren, Rosenthal and Wintrobe's Theorem}

In this section, we show a proof of the following theorem

Theorem ([7, Theorem 6.2]). If $\varphi$ is a hyperbolic disc automorphism and $\lambda$ is in the interior of the spectrum of $C_{\varphi}$, then $C_{\varphi}-\lambda I$ is universal.

Proof. Since every operator similar to a universal operator is universal, it suffices to consider a hyperbolic disc automorphism with fixed points \pm 1 . In particular, for $t_{0}>0$ we may consider

$$
\varphi_{t_{0}}(z)=\frac{\left(1+e^{-t_{0}}\right) z+\left(1-e^{-t_{0}}\right)}{\left(1-e^{-t_{0}}\right) z+\left(1+e^{-t_{0}}\right)}
$$

which has fixed points at 1 and -1 and derivatives at these fixed points $e^{-t_{0}}$ and $e^{t_{0}}$.

Let $\lambda$ in the interior of the spectrum. Then $\lambda \in \sigma_{p}\left(C_{\varphi_{t_{0}}}\right)$ (see [3], for instance), and therefore $e^{-t_{0} / 2}<|\lambda|<e^{t_{0} / 2}$. By (iii) in [4, Theorem 5] stated above, $C_{\varphi_{t_{0}}}-\lambda I$ is similar to $T_{\psi_{t_{0}}}^{*}-\lambda I=T_{\psi_{t_{0}}-\bar{\lambda}}^{*}$. So, we are reduced to show that $T_{\psi_{t_{0}}-\bar{\lambda}}^{*}$ satisfies Caradus Theorem.

Observe that the kernel of $T_{\psi_{t_{0}}-\bar{\lambda}}^{*}$ is infinite dimensional since $e^{-t_{0} / 2}<|\lambda|<e^{t_{0} / 2}$, the map $\psi_{t_{0}}$ takes any point in the annulus infinitely many times and the corresponding reproducing kernels are linearly independent. So, hypotheses (i) in Caradus Theorem is automatically satisfied.

In order to show that condition (ii) in Caradus Theorem is satisfied, observe that $\psi_{t_{0}}-\bar{\lambda}$ is a bounded analytic function in $\mathbb{D}$ and there exists $\ell>0$ so that

$$
\left|\psi_{t_{0}}\left(e^{i \theta}\right)-\bar{\lambda}\right| \geq \ell
$$

almost everywhere on the unit circle $\partial \mathbb{D}$. Indeed, one may take $\ell$ the distance from $\lambda$ to the boundary of the spectrum of $T_{\psi_{t_{0}}}^{*}$, i. e. , $\partial \sigma\left(T_{\psi_{t_{0}}}\right)=\left\{|z|=e^{-t_{0} / 2}\right\} \cup\left\{|z|=e^{t_{0} / 2}\right\}$. Obviously, such a distance is strictly positive because $\lambda$ belongs to the interior of the spectrum.

Then $1 /\left(\psi_{t_{0}}-\bar{\lambda}\right)$ is a bounded function in $\partial \mathbb{D}$; and therefore the (non-analytic) Toeplitz operator $T_{1 /\left(\psi_{t_{0}}-\bar{\lambda}\right)}$ is a left inverse for the analytic Toeplitz operator $T_{\psi_{t_{0}}-\bar{\lambda}}$. Hence, $T_{\psi_{t_{0}}-\bar{\lambda}}^{*}$ has a right inverse, and therefore is onto. So, $T_{\psi_{t_{0}}-\bar{\lambda}}^{*}$ is universal because of Caradus Theorem; which concludes the proof. 
Remark 2.1. Let us point out that the argument addressed to prove condition (ii) in Caradus Theorem is a particular instance of a more general result: If $f$ is a bounded analytic function in $\mathbb{D}$ and there is $\ell>0$ so that $\left|f\left(e^{i \theta}\right)\right| \geq \ell$ almost everywhere on the unit circle, then $1 / f$ is in $L^{\infty}(\partial \mathbb{D})$ and the (non-analytic) Toeplitz operator $T_{1 / f}$ is a left inverse for the analytic Toeplitz operator $T_{f}$. (See [5, Lemma 3], for instance).

Finally, let us remark that the proof presented here of [7, Theorem 6.2], avoiding to make use of the fact that Blaschke products induce isometric multiplication operators in $H^{2}$, might be extended to other spaces of analytic functions to exhibit concrete examples of universal operators.

\section{REFERENCES}

[1] S. R. Caradus, Universal operators and invariant subspaces, Proc. Amer. Math. Soc. 23(1969), 526-527.

[2] I. Chalendar and J. R. Partington, Modern Approaches to the Invariant Subspace Problem, Cambridge University Press, 2011.

[3] C. C. Cowen and B. D. MacCluer Composition Operators on Spaces of Analytic Functions, CRC Press, 1995.

[4] C. C. Cowen and E. A. Gallardo-Gutiérrez, Unitary equivalence of one-parameter groups of Toeplitz and composition operators, J. Functional Analysis 261(2011), 2641-2655.

[5] C. C. Cowen and E. A. Gallardo-Gutiérrez, Consequences of Universality Among Toeplitz Operators, J. Math. Anal. Appl. 432(2015), 484-503.

[6] E. A. Nordgren, P. Rosenthal, and F. S. Wintrobe, Composition operators and the invariant subspace problem, C. R. Mat. Rep. Acad. Sci. Canada, 6(1984), 279-282.

[7] E. Nordgren, P. Rosenthal and F. S. Wintrobe, Invertible composition operators on $H^{p}$, J. Functional Analysis, 73, (1987), no. 2, 324-344.

[8] J. R. Partington, Personal Communication. See http://www1.maths.leeds.ac.uk/ pmt6jrp/maisp_errata.pdf and the comments concerning [2, Chapter 8].

[9] G.-C. Rota, On models for linear operators, Comm. Pure Appl. Math. 13(1960), 469-472.

Department of Mathematical Sciences,

Indiana University-PuRdue University Indianapolis,

INDIANAPOLIS, IN 46202, USA

E-mail address: ccowen@iupui.edu

Universidad Complutense de MAdRId e ICMAT

Departamento de Análisis Matemático,

Facultad de Ciencias Matemáticas,

Plaza de Ciencias 3

28040, MADRID (SPAIN)

E-mail address: eva.gallardo@mat.ucm.es 